\title{
Nonlinear Adaptive Bottom-Following Control of an AUV in the Presence of Ocean Currents
}

\author{
Xue Yang \\ College of Science and Information, Qingdao Agricultural University, \\ Qingdao 266109, China \\ yangxue_qau@126.com
}

\begin{abstract}
This paper addresses the bottom-following control problem of an underactuated autonomous underwater vehicle (AUV) in the presence of ocean currents and model parameters uncertainties. The Serret-Frenet frame is used to describe the bottom following of the AUV. An extra degree of freedom for controller design is introduced. The extra degree of freedom makes the virtual AUV can regulate its velocity along with the real AUV. Based on Lyapunov theory and backstepping techniques, a nonlinear adaptive control law is derived. The control law yields convergence of the AUV to the desired path asymptotically. Simulations results show the effectiveness and robustness of the derived controllers.
\end{abstract}

Keywords: $A U V$, bottom-following, adaptive control, Serret-Frenet frame

\section{Introduction}

In recent years, autonomous underwater vehicles (AUVs) have great advantages in the area of oceanic research [1]. AUVs should have a bottom navigation ability to follow the bottom profile at a constant altitude as a basic feature for successful undersea search and survey, maritime reconnaissance, communication/navigation aids and tracking and trailing in uncharted shallow water [2]. This type of AUV considered in this work is equipped with two identical back thrusters mounted symmetrically with respect to its longitudinal axis. Especially, the AUV is underactuated since it lacks any vertical thruster. In addition, AUVs' kinematic and dynamic models are highly nonlinear and coupled, making control design a hard task [3].

Path following control is important in the field of AUV control. It refers to the case where an AUV follows a predefined path without any temporal specifications. Some researchers have studied the problem in recent years by using different techniques. The problem of following straight lines by using input-output linearization and sliding mode control was addressed [4]. The experimental tracking results for a model ship using Lyapunov-based controllers were presented [5,6]. Two tracking solutions for a surface vessel were proposed, based on Lyapunov's direct method and passivity approach [7]. A path following controller to follow straight lines based on cascade approach and feedback linearization techniques was designed [8]. To follow both straight line and curve, the Serret-Frenet frame was used to study the path following control [9-11]. As a kind of special path following, the bottom-following of AUVs has been studied. A bottomfollowing controller was designed for autonomous underwater vehicles (AUVs) that take explicitly into account the bathymetric characteristics ahead of the vehicle measured by two echo sounders [12]. An increment feedback control method based on nonlinear iterative sliding mode control was presented for bottom-following, and the problem of chattering by the hydroplane is circumvented [13]. The Takagi-Sugeno decision approach was applied to the bottom-following control that is discomposed into forward speed control and depth control [14]. 
In practice, an AUV must ofen operate in the presence of ocean currents and the AUV model parameters are not known precisely. In this paper, the bottom following error coordinate is built on a Serret-Frenet frame. Controller design relies heavily on Lyapunov method and backstepping techniques. Parameter uncertainties are dealt with in an adaptive framework by augmenting Lyapunov candidate functions with terms that are quadratic in the parameter errors. This design procedure effectively creates an extra degree of freedom that can then be explored to avoid the singularities that occur when the distance to path is not well defined. The pitch velocity persistent excitation conditions are not required. The resulting control strategy yields global convergence of the actual path of the AUV to the desired path.

The organization of the paper is as follows. Section 2 presents the AUV model and the formulation of the bottom following. Adaptive control laws for the AUV in the presence of ocean currents and parametric model uncertainties are derived in Section 3. Section 4 presents the simulation and experimental studies, including the AUV that is to track a straight line and a curve. Finally, we make a brief conclusion on the paper in Section 5.

\section{Problem Formulation}

This section describes the kinematic and dynamic equations of motion of the AUV depicted in Figure 1 in the vertical plane in the presence of ocean currents and formulates the problem of steering it along a desired path. The desired path is identical with the path that is formed from the bottom profile and has equivalent altitude with the bottom profile path. The control inputs are the thruster surge force and the thruster pitch torque. The AUV has no verical thruster, so the AUV is underactuated.

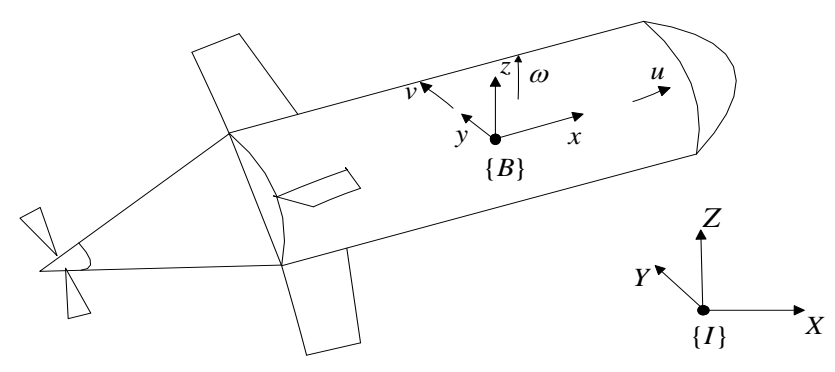

Figure 1. The Underactuated AUV Model

\subsection{Vehicle Modeling}

Following standard practice, the general kinematic and dynamic equations of the AUV can be developed using a global coordinate frame $\{I\}$ and a body-fixed coordinate frame $\{B\}$, as depicted in Figure 2. Let $Q$ denote the center of mass of the AUV and let $m=(x, 0, z)^{T}$ be the position of $Q$ in $\{I\}$. Let $\theta_{B}$ denote the yaw angle and $V_{t}=(u, 0, \omega)^{T}$ be the velocity of $Q$ in $\{I\}$ expressed in $\{B\}$, where $u$ and $\omega$ are the surge and heave velocities, respectively. 


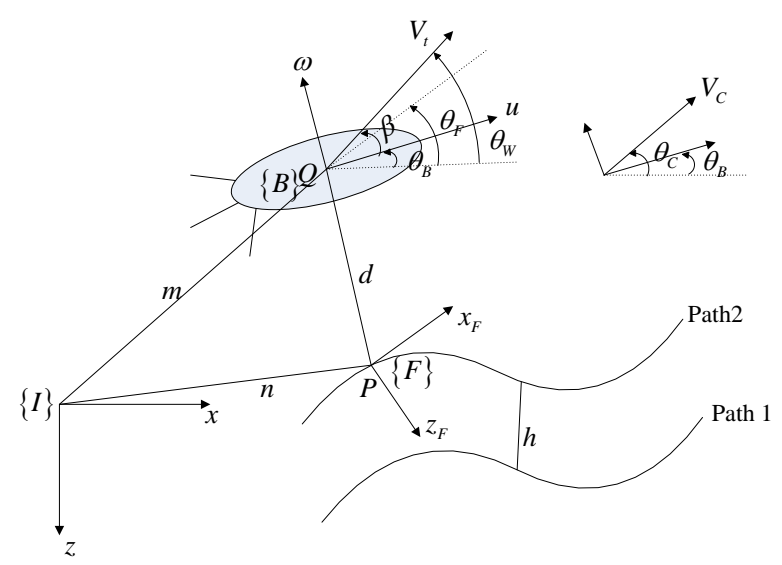

Figure 2. The Schematic Diagram of Bottom Following of the AUV

In the vertical plane, the kinematic equations of the AUV in the presence of ocean currents can be written as

$$
\left\{\begin{array}{l}
\dot{x}=u \cos \theta_{B}+\omega \sin \theta_{B}+V_{c} \cos \theta_{C} \\
\dot{z}=-u \sin \theta_{B}+\omega \cos \theta_{B}+V_{c} \sin \theta_{C} \\
\dot{\theta}_{B}=q
\end{array}\right.
$$

where $q$ is the angular velocity of the AUV, $V_{c}$ and $\theta_{C}$ denote the velocity and the angle of ocean currents in $\{I\}$, respectively. Assume $u$ never equal to zero, and define the side-slip angle $\beta=\arctan (\omega / u)$. The above equation can be rewritten to yield

$$
\left\{\begin{array}{l}
\dot{x}=V_{t} \cos \theta_{W}+V_{c} \cos \theta_{C} \\
\dot{z}=V_{t} \sin \theta_{W}+V_{c} \sin \theta_{C} \\
\dot{\theta}_{W}=q+\dot{\beta}
\end{array}\right.
$$

where $\theta_{W}=\theta_{B}+\beta, V_{t}$ is the total velocity and $V_{t}=\sqrt{u^{2}+\omega^{2}}$.

Neglecting the equations in sway, roll and yaw, the simplified equations for surge, heave and pitch can be written as [15]

$$
\left\{\begin{array}{l}
X=m_{u} \dot{u}-d_{u} \\
0=m_{\omega} \dot{\omega}+m_{u q} u q-d_{\omega} \\
M=m_{q} \dot{q}-d_{q}
\end{array}\right.
$$

where

$$
\begin{gathered}
m_{u}=m-X_{\dot{u}}, m_{\omega}=m-Z_{\dot{\omega}}, m_{q}=I_{y}-M_{\dot{q}}, m_{\omega q}=-m+X_{\omega q}, m_{u q}=m+Z_{u q}, \\
d_{u}=X_{q q} q^{2}+X_{u u} u^{2}+X_{\omega \omega} \omega^{2}+m_{\omega q} \omega q, d_{\omega}=Z_{\omega|\omega|} \omega|\omega|+Z_{\omega \omega} \omega^{2}, \\
d_{q}=M_{q|q|} q|q|+M_{u q} u q+M_{\omega \omega} \omega^{2}+M_{u \omega} u \omega+M_{u|\omega|} u|\omega|+M_{\omega|q|} \omega|q|+M_{\omega|\omega|} \omega|\omega| .
\end{gathered}
$$


$m$ and $I_{y}$ denote the mass and moment of inertia of the AUV, respectively. $m_{(.)}$is mass and hydrodynamic added mass term. $X_{(.)}, Z_{(.)}$and $M_{(.)}$are classical hydrodynamic derivatives, and $d_{(.)}$represents the hydrodynamic damping term. $(X, M)^{T}$ denotes the input vector of force and torque that is applied to the AUV. In the equations, and for clarity of presentation, it is assumed that the AUV is neutrally buoyant and that the centre of buoyancy coincides with the centre of gravity.

\subsection{Bottom-Following: Error Coordinates}

Consider Figure 2, where $P$ is an arbitrary point on the Path 2. Associated with $P$, consider the corresponding Serret-Frenet frame $\{F\}$. The signed curvilinear abscissa of $P$ along the Path 2 is denoted $s$. Clearly, $Q$ can either be expressed as $m=(x, 0, z)^{T}$ in $\{I\}$ or as $d=\left(x_{F}, 0, z_{F}\right)^{T}$ in $\{F\}, d$ is the vector from $P$ to $Q$. Let

$$
R=\left(\begin{array}{ccc}
\cos \theta_{F} & 0 & -\sin \theta_{F} \\
0 & 1 & 0 \\
\sin \theta_{F} & 0 & \cos \theta_{F}
\end{array}\right)
$$

be the rotation matrix from $\{I\}$ to $\{F\}$, parameterized locally by the angle $\theta_{F}$. Define $q_{F}=\dot{\theta}_{F}$, then

$$
\left\{\begin{array}{l}
q_{F}=\dot{\theta}_{F}=c_{c}(s) \dot{S} \\
\dot{c}_{c}(s)=g_{c}(s) \dot{s}
\end{array}\right.
$$

where $c_{c}(s)$ and $g_{c}(s)$ denote the path curvature and its derivative, respectively.

It is also straightforward to compute the velocity of $Q$ in $\{I\}$ expressed in $\{F\}$ as

$$
R\left(\frac{d m}{d t}\right)_{I}=\left(\frac{d n}{d t}\right)_{F}+\left(\frac{d d}{d t}\right)_{F}+q_{F} \times d
$$

Using the relations

$$
\left(\frac{d m}{d t}\right)_{I}=(\dot{x}, 0, \dot{z})^{T},\left(\frac{d n}{d t}\right)_{F}=(\dot{s}, 0,0)^{T},\left(\frac{d d}{d t}\right)_{F}=\left(\dot{x}_{F}, 0, \dot{z}_{F}\right)^{T}
$$

and

$$
q_{F} \times d=\left(c_{c}(s) \dot{s} z_{F}, 0,-c_{c}(s) \dot{s} x_{F}\right)^{T}
$$

Equation (5) can be rewritten as

$$
\left\{\begin{array}{l}
\dot{x}_{F}=\dot{x} \cos \theta_{F}-\dot{z} \sin \theta_{F}-\dot{s}-c_{c}(s) \dot{s} z_{F} \\
\dot{z}_{F}=\dot{x} \sin \theta_{F}+\dot{z} \cos \theta_{F}+c_{c}(s) \dot{s} x_{F}
\end{array}\right.
$$

Replacing the top two equations of (2) in (6) and introducing the variables $\theta=\theta_{W}+\theta_{F}$ and $\theta_{C F}=\theta_{C}+\theta_{F}$, the kinematic bottom-following error model of the AUV in the presence of ocean currents is given as 


$$
\left\{\begin{array}{l}
\dot{x}_{F}=-c_{c}(s) \dot{s} z_{F}+V_{t} \cos \theta-\dot{s}+V_{c} \cos \theta_{C F} \\
\dot{z}_{F}=c_{c}(s) \dot{s} x_{F}+V_{t} \sin \theta+V_{c} \sin \theta_{C F} \\
\dot{\theta}=q+\dot{\beta}+c_{c}(s) \dot{s}
\end{array}\right.
$$

With the above notations, the bottom-following problem of the AUV can be formulated as follows:

Consider the AUV model given by (1) and (3). Given a desired path having identical altitude with the bottom profile path to be followed and a desired profile $u_{d}>u_{\min }>0$ for the surge velocity $u$, derive control laws for the force $X$, torque $M$, and rate of evolution $\dot{s}$ of the curvilinear abscissa $s$ of the virtual target point $P$ along the path so that $x_{F}, z_{F}, \theta$ and $u-u_{d}$ tend to zero asymptotically in the presence of constant known ocean currents disturbance and parametric model uncertainties.

\section{Adaptive Bottom-Following Controller Design}

This section proposes a nonlinear adaptive control law to regulate the underactuated AUV along a desired bottom path in the presence of constant known ocean currents and parametric model uncertainties. The design of controller is implemented in three steps. The first step yields a kinematic controller by adopting $q$ as a "virtual" control input, and by assuming that the actual surge velocity equals with the desired velocity $u_{d}$. The second step addresses control laws for the "real" input $X$ and $M$. The third step applies adaptive control law to ensure robustness against uncertainties in the model parameters.

\subsection{Kinematic Controller}

This section derives a kinematic controller for the AUV. We let

$$
\delta\left(z_{F}\right)=\arcsin \left(\frac{-k_{0} z_{F}}{z_{F}^{2}+\varepsilon}\right)
$$

be a desired approach angle, where $k_{0}>0$ and $\varepsilon>0$. The approach angle is instrumental in shaping transient maneuvers during the path approach phase.

Theorem 1. Let the approach angle $\delta\left(z_{F}\right)$ be defined as in (8). Suppose that the path to be followed is parameterized by its curvilinear abscissa $s$, and the velocity $V_{C}$ and the orientation angle $\theta_{C}$ of the ocean currents are available for AUV sensors. Consider the kinematic model of the AUV described in (1) and the error model (7) with the control law

$$
\left\{\begin{array}{l}
\dot{s}=k_{2} x_{F}+V_{t} \cos \theta+V_{c} \cos \theta_{C F} \\
q=-\dot{\beta}-c_{c}(s) \dot{s}+\dot{\delta}-\frac{1}{k_{1}} z_{F} V_{t} \frac{\sin \theta-\sin \delta}{\theta-\delta}-\frac{1}{k_{1}} z_{F} V_{C} \frac{\sin \theta_{C F}-\sin \delta}{\theta-\delta}-k_{3}(\theta-\delta)
\end{array}\right.
$$

where $k_{i}>0 \quad(i=1,2,3)$. For any initial conditions, $x_{F}, z_{F}$ and $\theta$ tend to zero asymptotically in the presence of constant known ocean current.

Proof. Let the AUV move with a constant surge velocity $u=u_{d}>0$. Consider the candidate Lyapunov function 


$$
V_{1}=\frac{1}{2}\left[x_{F}^{2}+z_{F}^{2}+k_{1}(\theta-\delta)^{2}\right]
$$

where $k_{1}$ is a positive constant, and compute its derivative, one gives

$$
\begin{aligned}
\dot{V}_{1}= & x_{F} \dot{x}_{F}+z_{F} \dot{z}_{F}+k_{1}(\theta-\delta)(\dot{\theta}-\dot{\delta}) \\
= & x_{F}\left(-\dot{s}-c_{c}(s) \dot{s} z_{F}+V_{t} \cos \theta+V_{C} \cos \theta_{C F}\right)+z_{F}\left(c_{c}(s) \dot{s} x_{F}+V_{t} \sin \theta+V_{C} \sin \theta_{C F}\right) \\
& +k_{1}(\theta-\delta)\left(q+\dot{\beta}+c_{c}(s) \dot{s}-\dot{\delta}\right) \\
= & x_{F}\left(-\dot{s}+V_{t} \cos \theta+V_{C} \cos \theta_{C F}\right)+z_{F} V_{t} \sin \delta+z_{F} V_{C} \sin \delta \\
& +k_{1}(\theta-\delta)\left(q+\dot{\beta}+c_{c}(s) \dot{s}-\dot{\delta}+\frac{1}{k_{1}} z_{F} V_{t} \frac{\sin \theta-\sin \delta}{\theta-\delta}+\frac{1}{k_{1}} z_{F} V_{C} \frac{\sin \theta_{C F}-\sin \delta}{\theta-\delta}\right)
\end{aligned}
$$

Let the ideal kinematic laws for $s$ and $q$ be defined as Equation (9), then (10) becomes

$$
\dot{V}_{1}=-\frac{1}{k_{1}}\left(V_{t}+V_{C}\right) \frac{k_{0} z_{F}^{2}}{z_{F}^{2}+\varepsilon}-k_{1} x_{F}^{2}-k_{1} k_{3}(\theta-\delta)^{2}<0
$$

it can conclude that $x_{F}, z_{F}, \theta$ and $u-u_{d}$ tend to zero asymptotically.

\subsection{Dynamic Controller}

The above control law is derived in the kinematic model of the AUV. This control law can be extended to the dynamic model using backstepping techniques.

Theorem 2. Let the approach angle $\delta\left(z_{F}\right)$ be defined as in (8). Suppose that the path to be followed is parameterized by its curvilinear abscissa $s$, and the velocity $V_{C}$ and the orientation angle $\theta_{C}$ of the ocean currents are available for AUV sensors. Consider the model of the AUV described in (1), (3) and the error model (7) with the control law

$$
\left\{\begin{array}{l}
X=m_{u} \dot{u}_{d}-k_{4}\left(u-u_{d}\right)-d_{u} \\
M=m_{q} \dot{q}_{d}-k_{1}(\theta-\delta)-k_{5}\left(q-q_{d}\right)-d_{q}
\end{array}\right.
$$

where $k_{i}>0(i=4,5)$, and

$$
\begin{gathered}
q_{d}=-\dot{\beta}-c_{c}(s) \dot{s}+\dot{\delta}-\frac{1}{k_{1}} z_{F} V_{t} \frac{\sin \theta-\sin \delta}{\theta-\delta}-\frac{1}{k_{1}} z_{F} V_{C} \frac{\sin \theta_{C F}-\sin \delta}{\theta-\delta}-k_{3}(\theta-\delta) \\
\dot{q}_{d}=\frac{m_{u q} u \dot{u} q+m_{u q} u^{2} \dot{q}-u \dot{d}_{\omega}+m_{\omega} \omega \ddot{u}}{m_{\omega} V_{t}^{2}}+\Theta \\
\Theta=\frac{2 \dot{V}_{t} \dot{\beta}}{V_{t}}+c_{c}(s) \ddot{s}+g_{c}(s) \dot{s}^{2}+\ddot{\delta}-\frac{A}{k_{1}}-\frac{B}{k_{1}}-k_{3}(\dot{\theta}-\dot{\delta})
\end{gathered}
$$




$$
\begin{gathered}
A=\dot{z}_{F} V_{t} \frac{\sin \theta-\sin \delta}{\theta-\delta}+z_{F} \dot{V}_{t} \frac{\sin \theta-\sin \delta}{\theta-\delta} \\
+z_{F} V_{t} \frac{(\dot{\theta} \cos \theta-\dot{\delta} \cos \delta)(\theta-\delta)-(\sin \theta-\sin \delta)(\dot{\theta}-\dot{\delta})}{(\theta-\delta)^{2}} \\
B=\dot{z}_{F} V_{t} \frac{\sin \theta_{C F}-\sin \delta}{\theta-\delta} \\
+z_{F} V_{C} \frac{\left(c_{c}(s) \dot{s} \cos \theta_{C F}-\dot{\delta} \cos \delta\right)(\theta-\delta)-\left(\sin \theta_{C F}-\sin \delta\right)\left(c_{c}(s) \dot{s}-\dot{\delta}\right)}{(\theta-\delta)^{2}}
\end{gathered}
$$

For any initial conditions, $x_{F}, z_{F}, \theta$ and $u-u_{d}$ tend to zero asymptotically in the presence of constant known ocean currents.

Proof. Introduce error variable

$$
z=\left(z_{1}, z_{2}\right)^{T}=\left(u-u_{d}, q-q_{d}\right)^{T}
$$

choose the candidate Lyapunov function

$$
V_{2}=V_{1}+\frac{1}{2} m_{u} z_{1}^{2}+\frac{1}{2} m_{q} z_{2}^{2}
$$

and compute its derivative

$$
\begin{aligned}
\dot{V}_{2}= & -\frac{1}{k_{1}}\left(V_{t}+V_{C}\right) \frac{k_{0} z_{F}^{2}}{z_{F}^{2}+\varepsilon}-k_{1} x_{F}^{2}-k_{1} k_{3}(\theta-\delta)^{2}+z_{1}\left(X+d_{u}-m_{u} \dot{u}_{d}\right) \\
& +z_{2}\left(M+d_{q}-m_{q} \dot{q}_{d}+k_{1}(\theta-\delta)\right)
\end{aligned}
$$

Let the laws for $X$ and $M$ be defined as Equation (11), then (13) becomes

$$
\dot{V}_{2}=-\frac{1}{k_{1}}\left(V_{t}+V_{C}\right) \frac{k_{0} z_{F}^{2}}{z_{F}^{2}+\varepsilon}-k_{1} x_{F}^{2}-k_{1} k_{3}(\theta-\delta)^{2}-k_{4} z_{1}^{2}-k_{5} z_{2}^{2} \leq 0
$$

so $x_{F}, z_{F}, \theta$ and $u-u_{d}$ tend to zero asymptotically.

An extra degree of freedom for controller design is introduced, i.e., the velocity of vertical AUV on the desired path. Actually, the virtual AUV can reduce the velocity of itself or even wait for the real AUV when the real AUV is behind to it. Naturally, the virtual AUV will increase its velocity to catch up with the real one till their velocities are identical. It can be seen that the virtual AUV can regulate its velocity along with the real AUV.

\subsection{Parameter Adaptation}

So far, it is assumed that the AUV model parameters are known precisely. This assumption is unrealistic. In this section, the control law is tackled to ensure robustness against uncertainties in the model parameters. Consider the set of parameters of the AUV model concatenated in the vector

$$
\begin{gathered}
\Xi=\left(m_{u}, m_{q}, m_{\omega q}, M_{u \omega}, M_{u|\omega|}, M_{\omega \omega}, M_{\omega|\omega|}, M_{u q}, M_{\omega|q|},\right. \\
\left.M_{q|q|}, X_{u u}, X_{\omega \omega}, X_{q q}, m_{q} m_{u q}, m_{q} Z_{\omega \omega}, m_{q} Z_{\omega|\omega|}\right)^{T}
\end{gathered}
$$


and define the parameter estimation error $\tilde{\Xi}$ as $\tilde{\Xi}=\Xi-\hat{\Xi}$, where $\hat{\Xi}$ denotes a nominal value of $\Xi$. Consider the augmented candidate Lyapunov function

$$
V_{3}=V_{2}+\frac{1}{2} \tilde{\Xi}^{T} \mathrm{H}^{-1} \tilde{\Xi}
$$

where $\mathrm{H}=\operatorname{diag}\left\{r_{1}, r_{2}, \cdots, r_{16}\right\}$, and $r_{i}>0, i=1,2, \cdots, 16$ are the adaptation gains.

Choose the control laws

$$
\left\{\begin{aligned}
X= & \hat{m}_{u} \dot{u}_{d}-\hat{X}_{q q} q^{2}-\hat{X}_{u u} u^{2}-\hat{X}_{\omega \omega} \omega^{2}-\hat{m}_{\omega q} \omega q-k_{4}\left(u-u_{d}\right) \\
M= & \hat{m}_{q} \hat{m}_{u q} \frac{u \dot{u} q+u^{2} \dot{q}}{m_{\omega} V_{t}^{2}}-\hat{m}_{q} \hat{Z}_{\omega \omega} \frac{2 u \omega \dot{\omega}}{m_{\omega} V_{t}^{2}}-\hat{m}_{q} \hat{Z}_{\omega|\omega|} \frac{u(\operatorname{sign}(\omega) \dot{\omega}+\dot{\omega}|\omega|)}{m_{\omega} V_{t}^{2}}-\hat{M}_{q|q|} q|q| \\
& -\hat{M}_{u q} u q-\hat{M}_{\omega \omega} \omega^{2}-\hat{M}_{u \omega} u \omega-\hat{M}_{u|\omega|} u|\omega|-\hat{M}_{\omega|q|} \omega|q|-\hat{M}_{\omega|\omega|} \omega|\omega|+\Omega
\end{aligned}\right.
$$

where

$$
\Omega=\frac{\omega \ddot{u}}{V_{t}^{2}}+\frac{2 \dot{V}_{t} \dot{\beta}}{V_{t}}-c_{c}(s) \ddot{s}-g_{c}(s) \dot{s}^{2}+\ddot{\delta}-\frac{A}{k_{1}}-\frac{B}{k_{1}}-k_{3}(\dot{\theta}-\dot{\delta})-k_{5}\left(q-q_{d}\right)
$$

then

$$
\dot{V}_{3}=-\frac{1}{k_{1}}\left(V_{t}+V_{C}\right) \frac{k_{0} z_{F}^{2}}{z_{F}^{2}+\varepsilon}-k_{1} x_{F}^{2}-k_{1} k_{3}(\theta-\delta)^{2}-k_{4} z_{1}^{2}-k_{5} z_{2}^{2}+\tilde{\Xi}^{T}\left(\Gamma-\mathrm{H}^{-1} \dot{\hat{\Xi}}\right)
$$

where $\Gamma$ is a diagonal matrix given by

$$
\begin{array}{r}
\Gamma=\operatorname{diag}\left\{-\dot{u}_{d} z_{1},-\dot{q}_{d} z_{2}, \omega q z_{1}, u \omega z_{2}, u|\omega| z_{2}, \omega^{2} z_{2}, \omega|\omega| z_{2}, u q z_{2}, \omega|q| z_{2}, q|q| z_{2},\right. \\
\left.u^{2} z_{1}, \omega^{2} z_{1}, q^{2} z_{1},-\frac{u \dot{u} q+u^{2} \dot{q}}{m_{\omega} V_{t}^{2}} z_{2}, \frac{2 u \omega \dot{\omega}}{m_{\omega} V_{t}^{2}} z_{2}, \frac{u(\operatorname{sign}(\omega) \dot{\omega}+|\omega| \dot{\omega})}{m_{\omega} V_{t}^{2}} z_{2}\right\}
\end{array}
$$

Choose the parameter adaptation law as

$$
\dot{\hat{\Xi}}=\mathrm{H} \Gamma
$$

to yield

$$
\dot{V}_{3}=-\frac{1}{k_{1}}\left(V_{t}+V_{C}\right) \frac{k_{0} z_{F}^{2}}{z_{F}^{2}+\varepsilon}-k_{1} x_{F}^{2}-k_{1} k_{3}(\theta-\delta)^{2}-k_{4} z_{1}^{2}-k_{5} z_{2}^{2} \leq 0
$$

The above results play an important role in the proof of the following theorem that deals with the AUV model parameter uncertainty.

Theorem 3. Consider the model of the AUV described in (1), (3) and the desired approach angle defined in (8), together with the control law (14) and the parameter adaptation law (15). Then $x_{F}, z_{F}, \theta$ and $u-u_{d}$ tend to zero asymptotically in the presence of constant known ocean currents and bounded plant parameter uncertainties in

$$
\begin{gathered}
\Xi=\left(m_{u}, m_{q}, m_{\omega q}, M_{u \omega}, M_{u|\omega|}, M_{\omega \omega}, M_{\omega|\omega|}, M_{u q}, M_{\omega|q|},\right. \\
\left.M_{q|q|}, X_{u u}, X_{\omega \omega}, X_{q q}, m_{q} m_{u q}, m_{q} Z_{\omega \omega}, m_{q} Z_{\omega|\omega|}\right)^{T}
\end{gathered}
$$




\section{Simulation Results}

This section illustrates the performance of the proposed bottom following control scheme in the presence of constant ocean current disturbance and parametric uncertainties. The desired path is characterized by its curvilinear abscissa $s$. In order to test the controller in a general case, we have chosen two types of paths in the following simulations. The desired path is set to keep a constant height $h$ to the bottom profile.

The desired velocity $u_{d}$ is set to $1 \mathrm{~m} / \mathrm{s}$. The velocity of ocean currents is $V_{C}=0.1 \mathrm{~m} / \mathrm{s}$, angle $\theta_{C}=\pi / 4 \mathrm{rad}$. The AUV parameters used in the simulations are:

Table 1. The AUV Parameters Known Precisely

\begin{tabular}{llll}
\hline Parameter & $m$ & $Z_{\dot{\omega}}$ & $m_{\omega}$ \\
\hline Value & 160 & -251.6 & 411.6 \\
\hline
\end{tabular}

The initial AUV parameters are set as following:

Table 2. The Initial AUV Parameters Estimated

\begin{tabular}{cccc}
\hline $\begin{array}{c}\text { Param } \\
\text { eter }\end{array}$ & $\begin{array}{c}\text { Initial } \\
\text { Value }\end{array}$ & $\begin{array}{c}\text { Param } \\
\text { eter }\end{array}$ & $\begin{array}{c}\text { Initial } \\
\text { Value }\end{array}$ \\
\hline$m_{u}$ & 190.64 & $M_{\omega|q|}$ & -12.87 \\
$m_{q}$ & 100 & $M_{q|q|}$ & -54.21 \\
$m_{\omega q}$ & -216.35 & $X_{u u}$ & 100 \\
$M_{u \omega}$ & -2.3 & $X_{\omega \omega}$ & 120 \\
$M_{u|\omega|}$ & -3.47 & $X_{q q}$ & 100 \\
$M_{\omega \omega}$ & 7.51 & $m_{u q}$ & 60 \\
$M_{\omega|\omega|}$ & -53.71 & $Z_{\omega \omega}$ & -51.34 \\
$M_{u q}$ & -21.4 & $Z_{\omega|\omega|}$ & -123.3 \\
\hline
\end{tabular}

\subsection{Slope Path}

In this section, we consider a polynomial parameterization of the form as

$$
\begin{cases}x(\mu)=\mu, z(\mu)=35 & 0 \leq \mu<20 \\ x(\mu)=\mu, z(\mu)=45-0.5 \mu & 20 \leq \mu<60 \\ x(\mu)=\mu, z(\mu)=15 & 60 \leq \mu<100 \\ x(\mu)=\mu, z(\mu)=-15+0.3 \mu & 100 \leq \mu<150 \\ x(\mu)=\mu, z(\mu)=45 & 150 \leq \mu<200\end{cases}
$$

The initial condition for the AUV is

$$
(x(0), z(0), \theta(0), u(0), \omega(0), q(0))^{T}=(0,40, \pi / 3,0.3,0,0)^{T}
$$

The desired approach angle is defined in (8), where $k_{0}=50, \varepsilon=100$. The control gains are selected as following: 


$$
k_{1}=10, k_{2}=30, k_{3}=1, k_{4}=10, k_{5}=1,
$$

and

$$
\mathrm{H}=\operatorname{diag}\{5,5,5,3,1,10,5,10,1,2,2,2,2,2,10,10\} \times 10^{2} .
$$

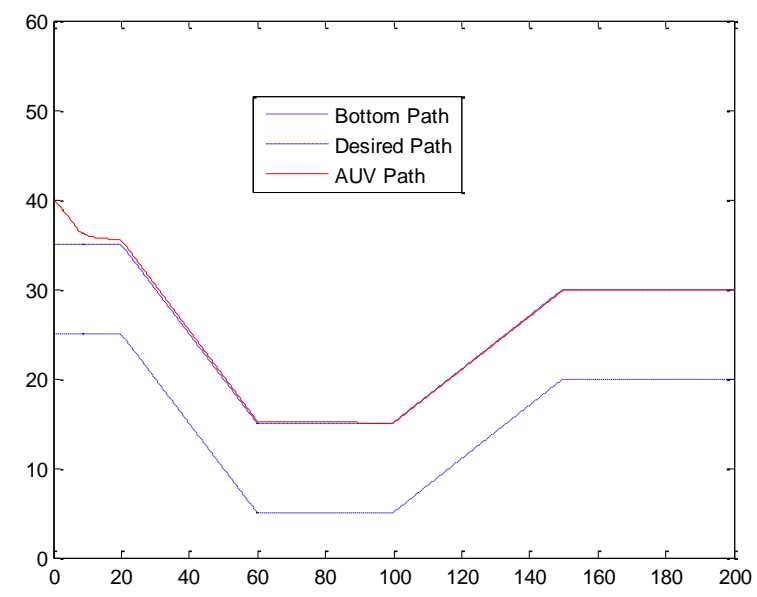

Figure 3. Simulation Resulting Paths of the AUV

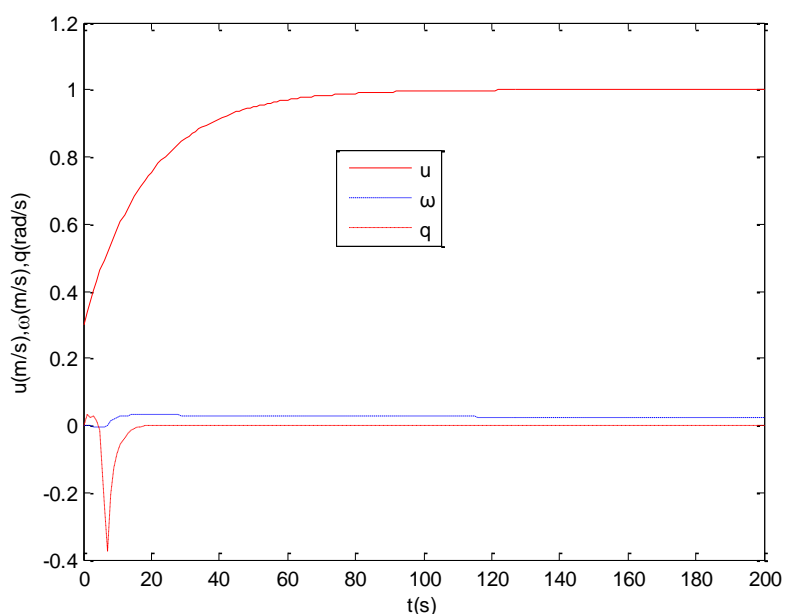

Figure 4. The Velocity and Angular Velocity of the AUV 


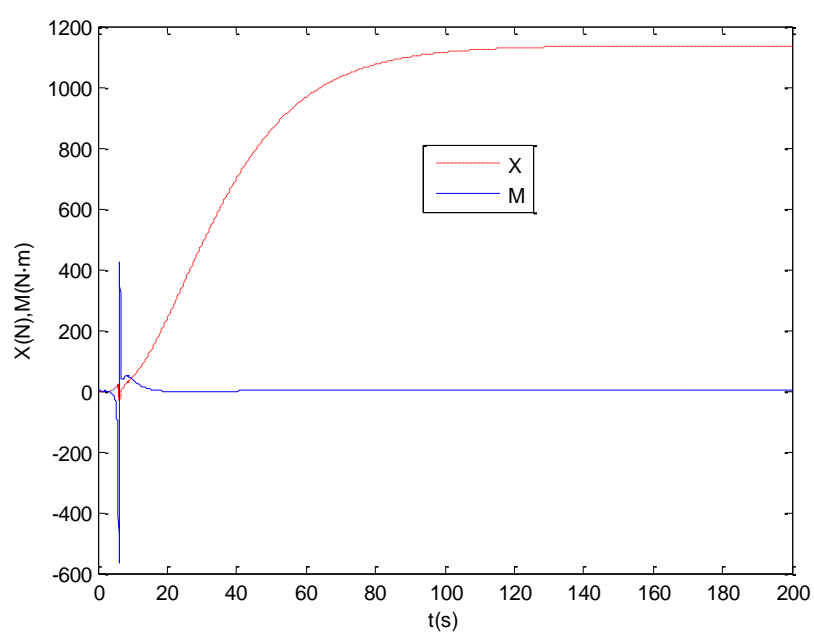

Figure 5. The Force and Torque of the AUV

\subsection{Curve Path}

In this section, we consider the path parameterized as following

$$
\left\{\begin{array}{l}
x(\mu)=\mu \\
z(\mu)=10 \sin (0.02 \mu)
\end{array}\right.
$$

The initial conditions for the AUV is

$$
(x(0), z(0), \theta(0), u(0), \omega(0), q(0))^{T}=(0,-15, \pi / 3,0.3,0,0)^{T}
$$

The desired approach angle is defined in (8), where $k_{0}=35, \varepsilon=100$. The control gains are selected as following:

$$
k_{1}=100, k_{2}=50, k_{3}=1, k_{4}=6, k_{5}=1,
$$

and

$$
\mathrm{H}=\operatorname{diag}\{50,5,50,3,10,10,50,10,10,2,3,3,3,30,10,10\} \times 10^{2} .
$$

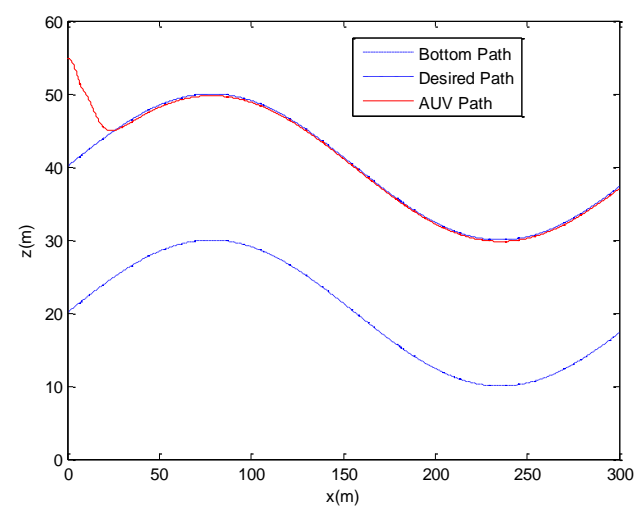

Figure 6. Simulation Resulting Paths of the AUV 


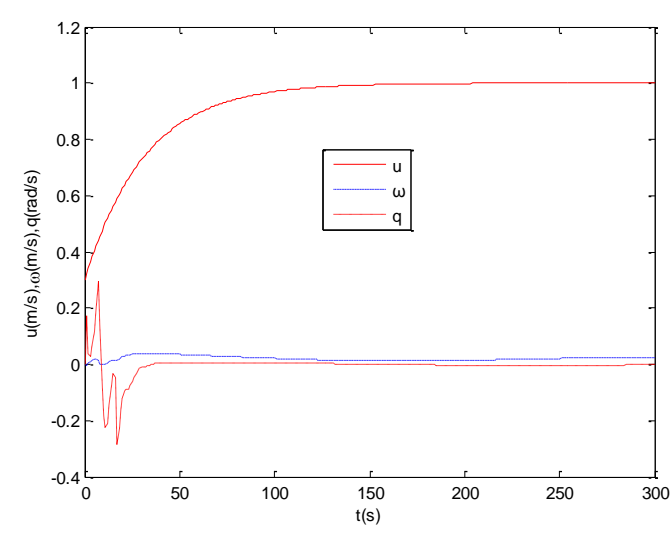

Figure 7. The Velocity and Angular Velocity of the AUV

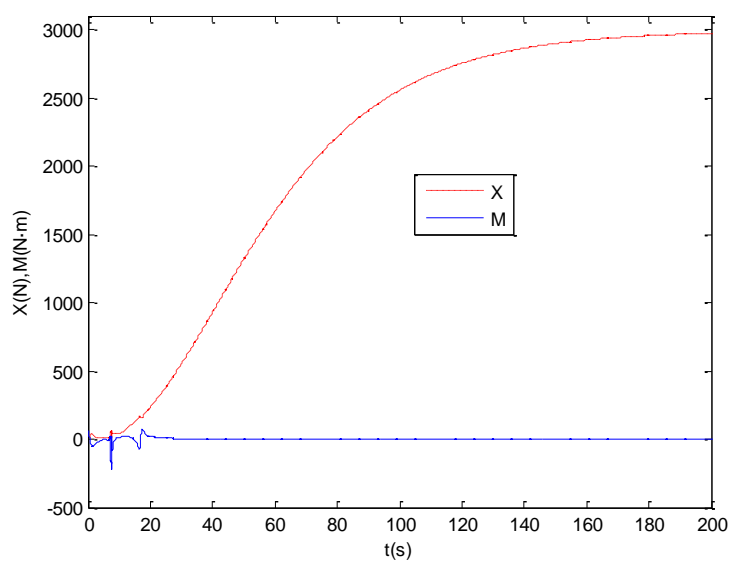

Figure 8. The Force and Torque of the AUV

Figure 3 and Figure 6 show good system reactions, i.e. the AUV converges to the desired path whether it is a straight line or a curve in the presence of known ocean currents and parametric model uncertainties. Velocity stabilization is shown in Figure 4 and Figure 7. It can be seen that the velocity $u$ tends to the desired velocity $u_{d}=1 \mathrm{~m} / \mathrm{s}$. Figure 5 and Figure 8 show the control force $X$ and the control torque $M$ needed for the bottom following control. After the AUV converges to the desired path, the control torque tends to zero. The constraint conditions of pitch velocity persistent excitation are not required.

\section{Conclusions}

In this paper, we have proposed an adaptive bottom-following control laws for the underactuated AUV in the presence of constant ocean currents and parameter uncertainties. An extra degree of freedom for controller design has been introduced so that the "virtual AUV" could regulate its velocity along with the "real AUV". Controller designs rely on backstepping techniques and Lyapunov theory. The proposed control laws can ensure robustness against uncertainties in the model parameters. Simulation results have demonstrated the validity of the designed bottom-following control scheme. 


\section{Acknowledgements}

This work was supported by Doctor Research Startup Foundation of Qingdao Agricultural University (1114341).

\section{References}

[1] J. H. Li and P. M. Lee, "Design of an adaptive nonlinear controller for depth control of an autonomous underwater vehicle", Ocean Engineering, 32 (7): 2165-2181 (2005).

[2] M. Caccia, R. Bono and G. Bruzzone, "Variable-configuration UUVs for marine science applications", IEEE Robotics and Automation Magazine, 6 (2): 22-32 (1999).

[3] F. Repoulias and E. Papadopoulos, "Planar trajectory planning and tracking control design for underactuated AUVs", Ocean Engineering, 34 (11): 1650-1667 (2007).

[4] R. J. Zhang, Y. B. Chen, Z. Q. Sun, F. C. Sun and H. Z. Xu, "Path control of a surface ship in restricted waters using sliding mode", IEEE Transactions on Control Systems Technology, 8 (4): 722-732 (2000).

[5] K. Y. Pettersen and H. Nijmeijer, "Underactuated ship tracking control: theory and experiments", International Journal of Control, 74 (14): 1435-1446 (2001).

[6] E. Lefeber, K. Y. Pettersen and H. Nijmeijer, "Tracking control of an underactuated ship", IEEE Transactions on Control Systems Technology, 11 (1): 52-61 (2003).

[7] Z. P. Jiang, "Global tracking control of underactuated ships by Lyapunov's direct method", Automatica, 38 (2): 301-309 (2002).

[8] K. Y. Pettersen and E. Lefeber, "Way-point tracking control of ships", Proceedings of IEEE Conference on Decision and Control, ( 2001) December 940-945; Orlando, USA.

[9] K. D. Do and J. Pan, "State and output feedback robust path-following controllers for underactuated ships using Serret-Frenet frame", Ocean Engineering, 31 (5): 587-613 (2004).

[10] L. Lapierre, D. Soetanto and A. Pascoal, "Nonsingular path following control of a unicycle in the presence of parametric modeling uncertainties", International Journal of Robust and Nonlinear Control, 16 (10): 485-503 (2006).

[11] L. Lapierre and D. Soetanto, "Nonlinear path-following control of an AUV", Ocean Engineering, 34 (11): 1734-1744 (2007).

[12] C. Silvestre, R. Cunha, N. Paulino and A. Pascoal, "A bottom-following preview controller for autonomous underwater", IEEE Transaction on Control Systems Technology, 17 (2): 257-266 (2009).

[13] X. Q. Bian, X. Q. Cheng, H. M. Jia, Z. P. Yan and L. J. Zhang, "A bottom-following controller for underactuated AUV based on iterative sliding and increment feedback", Control and Decision, 26 (2): 289-292+296 (2011).

[14] Y. M. Li, L. Wan, Y. S. Sun and G. C. Zhang, "Attitude information fusion and bottom-following control for underactuated underwater vehicle", Control Theory and Applications, 30 (1): 118-122 (2013).

[15] T. I. Fossen, "Guidance and control of ocean vehicles", Wiley: New York (1994).

\section{Author}

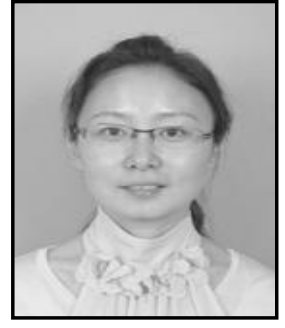

Xue Yang She received her doctor degree form Ocean University of China in 2014. Her research interest covers autonomous robots, nonlinear system control. 
International Journal of Control and Automation Vol.8, No.11 (2015) 